
\title{
$\begin{array}{ll}\text { Research Square } & \begin{array}{l}\text { Preprints are preliminary reports that have not undergone peer review. } \\ \text { They should not be considered conclusive, used to inform clinical practice, } \\ \text { or referenced by the media as validated information. }\end{array}\end{array}$
}

\section{Association of Myocardial Fibrosis Detected by Late Gadolinium-Enhanced MRI with Clinical Outcomes of Patients with Diabetes: A Systematic Review and Meta-Analysis}

Zhi Yang

Sichuan University West China Second University Hospital

Rong Xu

Sichuan University West China Second University Hospital

Jia-rong Wang

Sichuan University West China Hospital

Hua-yan Xu

Sichuan University West China Second University Hospital

Hang Fu

Sichuan University West China Second University Hospital

Ling-jun Xie

Sichuan University West China Second University Hospital

Meng-xi Yang

Sichuan University West China Second University Hospital

Lu Zhang

Sichuan University West China Second University Hospital

Ling-yi Wen

Sichuan University West China Second University Hospital

Hui Liu

Sichuan University West China Second University Hospital

Hong Li

Sichuan University West China Second University Hospital

Zhi-gang Yang

Sichuan University West China Hospital

Ying-kun Guo ( $\sim$ gykpanda@163.com )

Sichuan University West China Second University Hospital https://orcid.org/0000-0001-8437-9887

\section{Original investigation}

Keywords: Myocardial fibrosis, Late gadolinium-enhanced magnetic resonance imaging, Diabetes, Meta-analysis, Systemic review

Posted Date: June 4th, 2020

DOI: https://doi.org/10.21203/rs.3.rs-30053/v1

License: @) (7) This work is licensed under a Creative Commons Attribution 4.0 International License. Read Full License

Version of Record: A version of this preprint was published at BMJ Open on January 1st, 2022. See the published version at https://doi.org/10.1136/bmjopen2021-055374 


\section{Abstract}

Background Prior studies demonstrated that myocardial fibrosis assessed by late gadolinium-enhanced (LGE) MRI is associated with an increased risk for major adverse cardiac and cerebrovascular events (MACCE) or major adverse cardiac events (MACE) in patients with diabetes. However, the results of these studies were controversial and limited. Therefore, we performed this meta-analysis assessing the associations of myocardial fibrosis detected by LGE with the risk of MACCE and MACE in patients with diabetes.

Methods We selected studies using MEDLINE, EMBASE and Cochrane by Ovid on December 2019. Prospective and retrospective studies that assessed the associations of myocardial fibrosis detected by LGE with the risk of MACCE or MACE in patients with diabetes with a disease duration of at least 12 months. Two independent reviewers performed the data extraction using a standardized form. Pooled hazard ratios (HRs) and $95 \%$ confidence intervals (Cls) were evaluated by a random-effects model.

Results Eight studies with 1121 patients were included in this meta-analysis, and follow-up of patients ranged from 17 to 70 months. The prevalence of LGE in the total sample was high, occurring in $38.09 \%$. The annualized event rates (AERs) for MACCE was $11.94 \%$ in patients with diabetes and LGE. The presence of myocardial fibrosis detected by LGE was associated with an increased risk for MACCE (HR: 2.58; 95\% Cl 1.42-4.71; P=0.002) and MACE (HR: 5.28; 95\%Cl 3.208.70; $\mathrm{P}=0.000$ ) in patients with diabetes. In a subgroup meta-analysis, ischemic fibrosis detected by LGE was associated with MACCE/MACE (HR 3.75, 95\%Cl 2.11-6.69; $\mathrm{P}=0.000)$ in patients with diabetes. In diabetic patients with preserved ejection fraction, the association between myocardial fibrosis detected by LGE and MACCE/MACE remained significant (HR: 4.02; $95 \% \mathrm{Cl} 2.22-7.25 ; \mathrm{P}=0.000)$. All of the meta-analyses showed no significant heterogeneity from random effects.

Conclusion This study demonstrated that myocardial fibrosis detected by LGE conferred an increase in the risk of MACCE/MACE in patients with diabetes and may be an imaging biomarker for risk stratification.

\section{Background}

Diabetes is becoming a global healthcare problem, and it is estimated that there will be 693 million individuals with diabetes by 2045[1]. Patients with diabetes have a higher prevalence of myocardial fibrosis than their nondiabetic counterparts as a result of microvascular and macrovascular dysfunction, even when asymptomatic[2-5]. Moreover, the presence of myocardial fibrosis is associated with diabetic cardiomyopathy[6-8]. In addition, myocardial fibrosis can increase the risk of left ventricular (LV) dysfunction and heart failure with preserved ejection fraction in patients with diabetes[9, 10]. Therefore, it is important to detect myocardial fibrosis by noninvasive imaging technology for risk stratification in the clinical routine.

Among detectors of myocardial fibrosis, late gadolinium-enhanced magnetic resonance imaging (LGE-MRI) is the most reliable tool for identifying and quantifying myocardial fibrosis in vivo[11-13]. Furthermore, LGE-MRI is noninvasive and can easily discriminate between ischemic and nonischemic fibrosis without ionizing radiation[3]. Furthermore, recent guidelines suggested that MRI may be considered an imaging technique for stratifying cardiovascular risk in patients with diabetes[14, 15]. This highlights the role of LGE-MRI in risk stratification of patients with diabetes.

Approximately $19 \%$ of asymptomatic patients with diabetes have myocardial fibrosis upon LGE-MRI[2]. Although several studies have demonstrated that myocardial fibrosis detected by LGE-MRI may predict major adverse cardiac events (MACE) in patients with diabetes, the prognostic value of myocardial fibrosis for major cardiac and cerebrovascular events (MACCE) is unclear[2, 3, 16-21]. In addition, most previous studies were single-center studies and have been limited by small numbers of events. Consequently, we performed a meta-analysis to assess the association of LV myocardial fibrosis detected by late gadolinium enhancement (LGE) with future MACCE and MACE in patients with diabetes.

\section{Methods}

This meta-analysis was performed in accordance with the guidelines of the Meta-analysis of Observational Studies in Epidemiology (MOOSE) statement[22, 23].

\section{Data Sources and Searches}

We searched the Ovid MEDLINE, Ovid EMBASE and Ovid Cochrane Library databases to find eligible studies in December 2019. The search strategy included the following keywords: "diabetes”, "diabetes mellitus”, “MR”, “cardiac magnetic resonance”, “CMR”, “gadolinium”, "LGE”, "prognosis”, "diagnosed”, “predictor”, and "death". The details of the search strategy used for Ovid are available in Supplemental Table S1. In addition, only articles published in peer-reviewed journals and in the English language were included.

\section{Study Selection}

All articles were independently screened by two reviewers using the following inclusion criteria, and any disagreement was resolved by consensus. The inclusion criteria were as follows: the design was prospective or retrospective cohort study; the populations were patients with diabetes, and exposure of myocardial fibrosis was detected by LGE-MRI; the outcomes used composite endpoints including all-cause mortality, cardiac and cerebrovascular disease, late coronary revascularization, and hospitalization for unstable angina; the study reported the hazard ratio (HR) and $95 \%$ confidence intervals (CI) and had $\geq 12$ months of follow-up. We excluded reviews, abstracts, animal studies, case reports, and cross-sectional studies. Additionally, if the cases were reported more 
than once, we included the study with the most comprehensive information. Moreover, to obtain eligible studies, two reviewers independently screened the title first, then the abstract, and finally the full text.

\section{Data Extraction and Quality Assessment}

We extracted the following demographic data from each included study: author, year of publication, sample size, study design, age, LGE status, follow-up duration, outcome, and HR $(95 \% \mathrm{Cl})$. Additionally, we extracted the adjustment HR if the study reported the HR with adjustment models.

All of the included studies were prospective or retrospective cohort designs, and we used the Newcastle Ottawa Scale (NOS) to judge the study quality, which is usually used for evaluating the quality of cohort studies in meta-analyses[24, 25]. The scale uses a maximum of 9 points involving 3 factors: patient selection ( 0 to 4 points), comparability ( 0 to 2 points), and outcome ( 0 to 3 points)[26]. We delimited the quality of studies as low ( 0 to 3 scores), moderate (4 to 6 scores), and high (7 to 9 scores).

\section{Data Synthesis and Analysis}

In this meta-analysis, the outcome measure was the occurrence of future adverse cardiac and/or cerebrovascular events among diabetes patients with LGE compared to those without LGE. We defined the primary endpoint as MACCE, including myocardial infarction (MI), all-cause mortality, coronary and carotid revascularization, heart failure, ventricular arrhythmias, unstable angina, cardiac and cerebrovascular death, and cerebrovascular disease. The secondary end points were MACE, including all-cause mortality, cardiac death, MI, heart failure, unstable angina, and ventricular arrhythmias. Additionally, the pattern of myocardial fibrosis was classified as ischemic fibrosis or nonischemic fibrosis as described previously[3].

We pooled the adjusted $\mathrm{HR}$ with its $95 \% \mathrm{Cl}$ using a random-effects model. In addition, we calculated the annualized event rates (AERs) by dividing the total events by the median follow-up periods. To analyze the heterogeneity of the included studies, we used forest plots and the $\mathrm{I}^{2}$ statistic[27]. We assigned $\mathrm{I}^{2}$ values of $0 \otimes 25 \%, \varangle 50 \%, \varangle 75 \%$ for low, medium, and high heterogeneity of studies, respectively. Considering the heterogeneity of the included studies, we conducted sensitivity analyses by omitting 1 article to assess the influence of a single study. In particular, subgroup analyses were performed by outcome and the pattern of myocardial fibrosis. Additionally, a funnel plot was used to assess the publication bias of the included studies[28]. The analyses were performed with Stata version 12 (StataCorp). P values were two sided, with a level of 0.05 considered significant.

\section{Results}

\section{Literature Search}

Based on the selection strategy, we found 2134 citations. Of these, 151 duplicate studies were excluded. After screening the title and abstract, 12 articles remained for assessment of the full text. Four studies[29-32] were excluded for the following reasons: studies without our outcome of interest, study populations did not meet our inclusion criteria, and studies did not report the HR. Ultimately, 8 studies[2, 3, 16-21] fulfilled our inclusion criteria and were included in this meta-analysis (Fig. 1).

\section{Study Characteristics}

In aggregate, 8 studies included a total of 1121 patients with diabetes (median age ranging from 52 to $67 ; 67 \%$ were men) who underwent LGE-MRI and whose follow-up ranged from 17 to 70 months. Across the 8 studies, 6 articles[2, 17-21] reported the duration of diabetes, and the mean duration of diabetes was 15 years. A total of 6 studies[2, 3, 16, 19-21] reported the LV ejection fraction, and the mean LV ejection fraction was $57.78 \%$. The presence of LGE was evaluated by visual analysis in 6 publications[2, 3, 18-21]. All of the included studies reported multiple clinical outcomes. The main characteristics of the included articles are shown in Table 1. 


\begin{tabular}{|c|c|c|c|c|c|c|c|c|c|c|c|c|c|}
\hline $\begin{array}{l}\text { First } \\
\text { Author, } \\
\text { Year }\end{array}$ & Journal & patients & Hblc, \% & $\begin{array}{l}\text { LGE } \\
\text { Defintion }\end{array}$ & $\begin{array}{l}\text { DM } \\
\text { (type) }\end{array}$ & $\begin{array}{l}\text { Mean age } \\
\text { (years) }\end{array}$ & $\begin{array}{l}\text { Duration } \\
\text { of } \\
\text { Diabetes } \\
\text { (years) }\end{array}$ & LVEF (\%) & $\begin{array}{l}\text { Follow- } \\
\text { up } \\
\text { duration } \\
\text { (months) }\end{array}$ & male & $\operatorname{LGE}(+)$ & $\begin{array}{l}\text { Total } \\
\text { events }\end{array}$ & $\begin{array}{l}\text { Adju } \\
\text { HR }\end{array}$ \\
\hline $\begin{array}{l}\text { Bertheau } \\
\text { RC,2016 }\end{array}$ & Eur Radiol & 61 & $\begin{array}{l}7.2 \\
(6.5- \\
7.9)\end{array}$ & visual & $\begin{array}{l}1 \text { and } \\
2\end{array}$ & $\begin{array}{l}67.5(56.7- \\
71.8)\end{array}$ & $\begin{array}{l}19(14- \\
28)\end{array}$ & $56(46-61)$ & $\begin{array}{l}70(57- \\
72)\end{array}$ & 31 & 17 & 8 & YES \\
\hline $\begin{array}{l}\text { Heydari } \\
\text { B,2016 }\end{array}$ & $\begin{array}{l}\text { Circ } \\
\text { Cardiovasc } \\
\text { Imaging }\end{array}$ & 173 & $7.9 \pm 1.8$ & $2 S D$ & NA & $61.7 \pm 11.9$ & NA & $51.8 \pm 17.6$ & $34.8 \pm 30$ & 109 & 88 & 21 & NO \\
\hline $\begin{array}{l}\text { Elliott } \\
\text { MD,2019 }\end{array}$ & $\begin{array}{l}\text { Diabetes } \\
\text { Care }\end{array}$ & 120 & NA & visual & $\begin{array}{l}1 \text { and } \\
2\end{array}$ & $52 \pm 13$ & $17 \pm 11$ & $63 \pm 9$ & $\begin{array}{l}46(33- \\
64)\end{array}$ & 65 & 23 & 19 & YES \\
\hline $\begin{array}{l}\text { Yoon } \\
\text { YE,2013 }\end{array}$ & Eur Radiol & 120 & $7.4 \pm 1.5$ & visual & 2 & $67 \pm 9$ & $11 \pm 11$ & $63 \pm 6$ & $\begin{array}{l}27(7- \\
112)\end{array}$ & 83 & 18 & 10 & NO \\
\hline $\begin{array}{l}\text { Giusca } \\
\text { S,2016 }\end{array}$ & $\begin{array}{l}\text { Eur Heart J } \\
\text { Cardiovasc } \\
\text { Imaging }\end{array}$ & 328 & NA & visual & NA & $67 \pm 11$ & NA & $57.7 \pm 11.6$ & $\begin{array}{l}35(23- \\
51.6)\end{array}$ & 250 & 176 & 26 & YES \\
\hline $\begin{array}{l}\text { Bamberg } \\
\mathrm{F}, 2013\end{array}$ & Radiology & 61 & $\begin{array}{l}7.2 \\
(6.5- \\
7.9)\end{array}$ & visual & $\begin{array}{l}1 \text { and } \\
2\end{array}$ & $\begin{array}{l}67.5(56.7- \\
71.8)\end{array}$ & $\begin{array}{l}19(14- \\
28)\end{array}$ & $56(46-61)$ & $\begin{array}{l}70(57- \\
72)\end{array}$ & 31 & 17 & 18 & YES \\
\hline $\begin{array}{l}\text { Kwong } \\
\text { RY,2008 }\end{array}$ & Circulation & 107 & $7.3 \pm 1.6$ & $2 S D$ & NA & $59 \pm 12$ & $10.7 \pm 8.5$ & NA & $17(6-57)$ & 67 & 30 & 38 & YES \\
\hline $\begin{array}{l}\text { Yoon } \\
\text { YE,2012 }\end{array}$ & Radiology & 151 & $7.4 \pm 1.6$ & visual & NA & $67 \pm 9$ & $14 \pm 11$ & NA & $\begin{array}{l}30(6- \\
103)\end{array}$ & 113 & 58 & 24 & NO \\
\hline
\end{tabular}

Columns represent $\mathrm{n}(\%)$ or mean \pm SD or median (IQR); DM, diabetes mellitus; LGE, late gadolinium enhancement; LVEF, left ventricular ejection fraction; NOS, reported; MACCE, major adverse cardiac and cerebrovascular events; MACE, major adverse cardiac events.

Table 1

Description of the Studies Included in the Meta-Analysis

Among the 8 selected studies, 6 studies[16-21] (75\%) were conducted in a single center (Germany, $n=2 ;$ USA, $n=2 ;$ Japan, $n=2$ ), and 2 studies[2, 3] were performed in multiple centers (USA, $n=1$; Europe, $n=1)$. Five articles[2, 3, 17, 20, 21] $(62.5 \%)$ reported adjusted HR. Six studies[2, 16, 18-21] reported patients with ischemic fibrosis, and the remaining 2 studies $[3,17]$ reported patients with ischemic and nonischemic fibrosis.

Of the 8 eligible studies, 7 received 7 to 9 scores, and the mean NOS score was 7.5. Overall, the aforementioned analysis showed that the included articles had high quality (Table 1). Among the identified studies, there was no risk of publication bias assessed by visual analysis of the funnel plot (Fig. 2).

\section{Prevalence of LGE and AERs}

Across the 8 studies, the prevalence of myocardial fibrosis detected by LGE ranged from $15 \%$ to $62 \%$, and the prevalence of LGE in the total sample was $38.09 \%(n=427)$. Furthermore, a total of 164 events occurred in the diabetes group $(n=1121)$ during the median follow-up of 3.4 years. Patients with diabetes had AERs for MACCE of $4.3 \%$. However, only 3 studies $[2,19,21]$ reported a total of 301 patients with diabetes. Among these patients, $19.27 \%$ ( $=58$ ) had myocardial fibrosis detected by LGE, with 27 events occurring over a median follow-up of 3.9 years. The AERs of patients with diabetes and LGE was $11.94 \%$.

\section{MACCE and MACE}

A total of 8 studies reported the outcome of MACCE or MACE, and the presence of myocardial fibrosis detected by LGE was a strong predictor of MACCE and MACE in patients with diabetes (random-effects HR 3.87, 95\% Cl 2.58-5.80; $\mathrm{P}=0.000$ ) (Fig. 3 ). Low heterogeneity $\left(\mathrm{I}^{2}=15.1 \%, \mathrm{P}=0.311\right)$ existed in the metaanalysis. In addition, sensitivity analysis performed by excluding 1 study each time found that the HR values were not significantly changed.

In the analysis of the outcome of MACCE, 3 articles[17, 20,21] were included in this subgroup meta-analysis, including 64 participants with LGE and 165 diabetes without LGE, with a total of 64 MACCE outcomes during the follow-up period. Myocardial fibrosis detected by LGE was associated with an increased risk of MACCE in patients with diabetes. The pooled random-effects HR was $2.58(95 \% \mathrm{Cl} 1.42-4.71 ; \mathrm{P}=0.002)$, with no evidence of heterogeneity $\left(\mathrm{I}^{2}=14.1 \%\right.$; $P=0.312)$ (Fig. 3).

To explore the association between myocardial fibrosis and the outcome of MACE in patients with diabetes, we included 5 articles[2, 3, 16, 18, 19] that provided a subgroup meta-analysis. The results showed that the presence of LGE in diabetes was associated with a significantly higher risk of MACE. As in the discovery analyses, the pooled HR was $5.28(95 \% \mathrm{Cl} 3.20-8.70 ; \mathrm{P}=0.000)$ with no significant heterogeneity $\left(I^{2}=0 \% ; P=0.643\right)$ from random effects $($ Fig. 3 ).

To further verify the robustness of the results, we grouped all included studies by adjusted or non-adjusted HR. In patients with diabetes, myocardial fibrosis detected by LGE was associated with an increased risk of MACCE and MACE in a subgroup meta-analysis with or without adjusted HR. The pooled HRs were 3.52 (random-effects, 95\% Cl 2.02-6.16; $\mathrm{I}^{2}=35.8 \%$ ) and 4.63 (random-effects, $95 \% \mathrm{Cl} 2.35-9.14 ; \mathrm{I}^{2}=0 \%$ ), respectively. There was no significant heterogeneity among the studies (Fig. 4). 
To evaluate the pattern of myocardial fibrosis effects, we further calculated a pooled HR by source of diabetes with different patterns of myocardial fibrosis. In patients with diabetes, ischemic fibrosis detected by LGE was significantly associated with increased MACCE and MACE (random-effects HR 3.75 , 95\% CI $2.11-6.69 ; I^{2}=38.3 \%$ ). Furthermore, all myocardial fibrosis detected by LGE in patients with diabetes may increase the risk of MACCE and MACE (randomeffects HR $4.27,95 \% \mathrm{Cl} 2.17-8.37 ; \mathrm{I}^{2}=0 \%$ ) (Fig. 5).

To confirm whether there were similar results in patients with preserved LV ejection fraction, we conducted a subgroup meta-analysis with 6 studies. Among individuals with diabetes and LV ejection fraction $>50 \%$, the presence of myocardial fibrosis assessed by LGE was significantly associated with MACCE and MACE. The pooled HR was $3.98(95 \% \mathrm{Cl} 2.22-7.25 ; \mathrm{P}=0.000)$ with random effects, and there was medium heterogeneity among the studies $\left(\mathrm{I}^{2}=37.9 \% ; \mathrm{P}=\right.$ 0.153) (Fig. 6).

\section{Discussion}

In this meta-analysis, the prevalence of myocardial fibrosis assessed by LGE was increased in patients with diabetes, occurring in $38.09 \%$ of them. In addition, the presence of myocardial fibrosis assessed by LGE was associated with an increased risk for MACCE and MACE, even when the LV ejection fraction persisted. Specifically, myocardial fibrosis detected by LGE has a higher predictive value for the occurrence of future MACE than MACCE in patients with diabetes. Furthermore, myocardial fibrosis by LGE may be an imaging biomarker for predicting adverse outcomes in patients with diabetes.

In our meta-analysis, the results supported previous studies showing that participants with diabetes have a higher presence of myocardial fibrosis detected by LGE. Importantly, in our included studies, the presence of myocardial fibrosis in symptomatic patients with diabetes was higher than that in asymptomatic patients with diabetes[2, 3, 17]. Given that more than $38.09 \%$ of patients with diabetes have myocardial fibrosis detected by LGE in our meta-analysis, it indicated that LGE is very important for screening myocardial fibrosis in diabetes. Current guidelines recommend that MRI may be a risk tool in asymptomatic patients with diabetes at moderate or high risk of cardiovascular disease[14]. However, the value of MRI in routine clinical stratification of cardiovascular risk is unclear. Notably, in our meta-analysis, focal myocardial fibrosis detected by LGE did seem to predict a higher occurrence MACCE/MACE in the future, and the AERs for MACCE/MACE in patients with diabetes and LGE was $11.94 \%$. Additionally, the presence of myocardial fibrosis indicated a 8 -fold higher risk for death/MI even in asymptomatic patients with diabetes[2]. It must be noted that other techniques, such as ECG, have lower accuracy and sensitivity for detecting myocardial fibrosis than LGE[33, 34]. Thus, this finding highlighted the value of LGE for screening for cardiovascular risk in patients with diabetes.

The risk of myocardial fibrosis in patients with diabetes is increased and likely multifactorial. First, patients with diabetes have a higher risk for coronary artery disease and myocardial dysfunction[35-37]. Moreover, hyperglycemic metabolism, microvascular disease, and cardiac autonomic neuropathy are involved in the mechanisms of myocardial fibrosis[4, 38,39]. However, many studies have shown that patients with diabetes have a high incidence of obesity, visceral fat, hyperlipidemia, and insulin resistance, which may impair myocardial function[6, 40,41]. Furthermore, the multiple risk factors described above should increase the myocardial fibrosis burden. In addition, myocardial fibrosis is widespread in subjects with diabetes and may be associated with a high risk for cardiovascular disease.

Although the focal myocardial fibrosis translates to an adverse outcome in future is not fully clear, several potential mechanisms may lead to MACCE/MACE. First, patients with diabetes are more inclined to develop myocardial fibrosis, and myocardial fibrosis is associated with ventricular arrhythmia and heart failure[3, 42-44]. Second, patients with diabetes and myocardial fibrosis usually have a greater burden of microvascular complications, such as myocardial ischemia, which confers an increased risk of MACCE/MACE[16, 45]. Additionally, the myocardial fibrosis detected by LGE, especially subendocardial fibrosis, indicates more severe coronary calcium and atherosclerotic disease, which denotes a higher risk of MACE[46, 47]. Furthermore, subjects with diabetes had higher LV and left atrial remodeling due to myocardial fibrosis $[9,43,48]$. For these reasons, the myocardial fibrosis detected by LGE indeed has clinical relevance.

As previously described, LGE-MRI has become a powerful noninvasive imaging method for the assessment of myocardial fibrosis[11]. Unfortunately, our metaanalysis demonstrated that the presence of myocardial fibrosis derived from LGE conferred an HR of 3.87 for future MACCE/MACE in individuals with diabetes, and the risk increased with ischemic myocardial fibrosis. It must be indicated that two studies[20,21] were included in our meta-analysis, which showed that ischemic myocardial fibrosis detected by LGE did not increase the rate of MACCE. This might be explained by the following reasons, such as limited patients and the patients having a high prevalence of cardiovascular disease. Indeed, detecting myocardial fibrosis can be used to clinically assess myocardial damage and to stratify cardiovascular risk in participants with diabetes. To date, only one study, which screened for asymptomatic diabetes by LGE, showed that diabetes with ischemic myocardial fibrosis conferred an 8-fold higher risk for all-cause mortality and MI[2]. The prevalence of myocardial fibrosis detected by LGE among patients with diabetes is higher than that among nondiabetic patients[3, 30]. Therefore, patients with diabetes and myocardial fibrosis might need aggressive management of cardiac and cerebrovascular risk factors.

However, our meta-analysis has some limitations. First, in our meta-analysis, 2 studies[20, 21] were from the same group of patients but reported different outcomes. However, when we excluded either of the above articles, the pooled HR and heterogeneity did not change significantly. Second, the incidence of myocardial fibrosis in patients with diabetes was not community-based epidemiology research. The prevalence of myocardial fibrosis, therefore, may be higher in this study, which pooled studies including high-risk or average-risk populations with diabetes. Third, a previous study found that women with diabetes had a higher risk for MACCE than men with diabetes[49]. However, this study was not designed to evaluate sex differences in the effect of myocardial fibrosis on MACCE/MACE in patients with diabetes. Fourth, most studies selected in this meta-analysis reported adjusted HRs, and various adjustments for adverse outcomes among the selected studies may affect the pooled results. However, the heterogeneity among the selected studies was low, and publication bias did not exist. This might strengthen the clinical meaning of the pooled result. Finally, the incremental value of diabetes duration to the prevalence and incidence of LGE was not revealed. However, diabetes duration plays a central role in the assessment of cardiovascular risk[14, 50]. Hence, a prospective study 
that evaluates the association between diabetes duration and myocardial fibrosis and determines the best time to screen myocardial fibrosis by LGE-CMR for risk stratification in patients with diabetes is needed.

\section{Conclusions}

In patients with diabetes, the presence of myocardial fibrosis detected by LGE-MRI was markedly associated with an important and increased risk of MACCE/MACE. Myocardial fibrosis may be a risk marker for improving risk stratification in patients with diabetes. This meta-analysis highlights the role of LGE-MRI in helping identify high-risk diabetic patients in routine clinical practice.

\section{Abbreviations}

LGE: late gadolinium-enhanced; MACCE: major adverse cardiac and cerebrovascular events; MACE: major adverse cardiac events; HRs: hazard ratios; Cis: confidence intervals; AERs: annualized event rates; LV: left ventricular; LGE-MRI: late gadolinium-enhanced magnetic resonance imaging; MOOSE: Metaanalysis of Observational Studies in Epidemiology; MI: myocardial infarction; NOS: Newcastle Ottawa Scale

\section{Declaration}

\section{Ethical approval and consent to participate}

Not applicable.

\section{Consent for publication}

Not applicable.

\section{Availability of data and materials}

Not applicable.

\section{Competing interests}

The authors declare that they have no competing of interests.

\section{Funding}

This work was supported by the National Natural Science Foundation of China (No. 81771887, 81771897, 81971586ه81901712); the Program for Young Scholars and Innovative Research Team in Sichuan Province (No. 2017TD0005) of China; and 1.3.5 project for disciplines of excellence, West China Hospital, Sichuan University (No.ZYGD18013).

\section{Authors' contributions}

Zhi Yang and Rong Xu conceived of this study, and participated in its design and coordination and drafted the manuscript, Zhi-gang Yang and Ying-kun Guo helped to draft the manuscript. Other participated in the design of the study and helped with the statistical analysis. All authors read and approved this manuscript.

\section{Acknowledgements}

Not applicable.

\section{Reference}

1. Cho NH, Shaw JE, Karuranga S, Huang Y, da Rocha Fernandes JD, Ohlrogge AW, et al. IDF Diabetes Atlas: Global estimates of diabetes prevalence for 2017 and projections for 2045. Diabetes research and clinical practice. 2018;138:271-81.

2. Elliott MD, Heitner JF, Kim H, Wu E, Parker MA, Lee DC, et al. Prevalence and prognosis of unrecognized myocardial infarction in asymptomatic patients with diabetes: A two-center study with up to 5 years of follow-up. Diabetes Care. 2019;42(7):1290-6.

3. Giusca S, Kelle S, Nagel E, Buss SJ, Voss A, Puntmann V, et al. Differences in the prognostic relevance of myocardial ischaemia and scar by cardiac magnetic resonance in patients with and without diabetes mellitus. European heart journal cardiovascular Imaging. 2016;17(7):812-20.

4. Jia G, Hill MA, Sowers JR. Diabetic Cardiomyopathy: An Update of Mechanisms Contributing to This Clinical Entity. Circulation research. 2018;122(4):62438. 
5. Russo I, Frangogiannis NG. Diabetes-associated cardiac fibrosis: Cellular effectors, molecular mechanisms and therapeutic opportunities. Journal of molecular and cellular cardiology. 2016;90:84-93.

6. Marwick TH, Ritchie R, Shaw JE, Kaye D. Implications of Underlying Mechanisms for the Recognition and Management of Diabetic Cardiomyopathy. Journal of the American College of Cardiology. 2018;71(3):339-51.

7. Adeghate E, Singh J. Structural changes in the myocardium during diabetes-induced cardiomyopathy. Heart failure reviews. 2014;19(1):15-23.

8. Jia G, Whaley-Connell A, Sowers JR. Diabetic cardiomyopathy: a hyperglycaemia- and insulin-resistance-induced heart disease. Diabetologia. 2018;61(1):21-8.

9. Storz C, Hetterich H, Lorbeer R, Heber SD, Schafnitzel A, Patscheider H, et al. Myocardial tissue characterization by contrast-enhanced cardiac magnetic resonance imaging in subjects with prediabetes, diabetes, and normal controls with preserved ejection fraction from the general population. European heart journal cardiovascular Imaging. 2018;19(6):701-8.

10. Armstrong AC, Ambale-Venkatesh B, Turkbey E, Donekal S, Chamera E, Backlund J-Y, et al. Association of Cardiovascular Risk Factors and Myocardial Fibrosis With Early Cardiac Dysfunction in Type 1 Diabetes: The Diabetes Control and Complications Trial/Epidemiology of Diabetes Interventions and Complications Study. Diabetes Care. 2017;40(3):405-11.

11. Mewton N, Liu CY, Croisille P, Bluemke D, Lima JAC. Assessment of myocardial fibrosis with cardiovascular magnetic resonance. Journal of the American College of Cardiology. 2011;57(8):891-903.

12. Hashimura H, Kimura F, Ishibashi-Ueda H, Morita Y, Higashi M, Nakano S, et al. Radiologic-Pathologic Correlation of Primary and Secondary Cardiomyopathies:MR Imaging and Histopathologic Findings in Hearts from Autopsy and Transplantation. Radiographics. 2017;37(3):719-36.

13. Iles LM, Ellims AH, Llewellyn H, Hare JL, Kaye DM, McLean CA, et al. Histological validation of cardiac magnetic resonance analysis of regional and diffuse interstitial myocardial fibrosis. European heart journal cardiovascular Imaging. 2015;16(1):14-22.

14. Cosentino F, Grant PJ, Aboyans V, Bailey CJ, Ceriello A, Delgado V, et al. 2019 ESC Guidelines on diabetes, pre-diabetes, and cardiovascular diseases developed in collaboration with the EASD. European heart journal. 2020;41(2):255-323.

15. Jensen MT, Fung K, Aung N, Sanghvi MM, Chadalavada S, Paiva JM, et al. Changes in Cardiac Morphology and Function in Individuals With Diabetes Mellitus: The UK Biobank Cardiovascular Magnetic Resonance Substudy. Circulation Cardiovascular imaging. 2019;12(9):e009476.

16. Heydari B, Juan YH, Liu H, Abbasi S, Shah R, Blankstein R, et al. Stress Perfusion Cardiac Magnetic Resonance Imaging Effectively Risk Stratifies Diabetic Patients with Suspected Myocardial Ischemia. Circulation: Cardiovascular Imaging. 2016;9(4).

17. Kwong RY, Sattar H, Wu H, Vorobiof G, Gandla V, Steel K, et al. Incidence and prognostic implication of unrecognized myocardial scar characterized by cardiac magnetic resonance in diabetic patients without clinical evidence of myocardial infarction. Circulation. 2008;118(10):1011-20.

18. Yoon YE, Kitagawa K, Kato S, Nakajima H, Kurita T, Ito M, et al. Prognostic significance of unrecognized myocardial infarction detected with MR imaging in patients with impaired fasting glucose compared with those with diabetes. Radiology. 2012;262(3):807-15.

19. Yoon YE, Kitagawa K, Kato S, Nakajima H, Kurita T, Dohi K, et al. Prognostic value of unrecognised myocardial infarction detected by late gadoliniumenhanced MRI in diabetic patients with normal global and regional left ventricular systolic function. European radiology. 2013;23(8):2101-8.

20. Bamberg F, Parhofer KG, Lochner E, Marcus RP, Theisen D, Findeisen HM, et al. Diabetes mellitus: Long-term prognostic value of whole-body MR imaging for the occurrence of cardiac and cerebrovascular events. Radiology. 2013;269(3):730-7.

21. Bertheau RC, Bamberg F, Lochner E, Findeisen HM, Parhofer KG, Kauczor HU, et al. Whole-Body MR Imaging Including Angiography: Predicting Recurrent Events in Diabetics. European radiology. 2016;26(5):1420-30.

22. Stroup DF, Berlin JA, Morton SC, Olkin I, Williamson GD, Rennie D, et al. Meta-analysis of observational studies in epidemiology: a proposal for reporting. Meta-analysis Of Observational Studies in Epidemiology (MOOSE) group. Jama. 2000;283(15):2008-12.

23. Hutton B, Salanti G, Caldwell DM, Chaimani A, Schmid CH, Cameron C, et al. The PRISMA extension statement for reporting of systematic reviews incorporating network meta-analyses of health care interventions: checklist and explanations. Ann Intern Med. 2015;162(11):777-84.

24. Stang A. Critical evaluation of the Newcastle-Ottawa scale for the assessment of the quality of nonrandomized studies in meta-analyses. European journal of epidemiology. 2010;25(9):603-5.

25. Zeng X, Zhang Y, Kwong JS, Zhang C, Li S, Sun F, et al. The methodological quality assessment tools for preclinical and clinical studies, systematic review and meta-analysis, and clinical practice guideline: a systematic review. Journal of evidence-based medicine. 2015;8(1):2-10.

26. Mantovani A, Byrne CD, Bonora E. Targher G. Nonalcoholic Fatty Liver Disease and Risk of Incident Type 2 Diabetes: A Meta-analysis. Diabetes Care. 2018;41(2):372-82.

27. Higgins JP, Thompson SG. Quantifying heterogeneity in a meta-analysis. Statistics in medicine. 2002;21(11):1539-58.

28. Liu JL. The role of the funnel plot in detecting publication and related biases in meta-analysis. Evidence-based dentistry. 2011;12(4):121-2.

29. Reinstadler SJ, Stiermaier T, Eitel C, Metzler B, de Waha S, Fuernau G, et al. Relationship between diabetes and ischaemic injury among patients with revascularized ST-elevation myocardial infarction. Diabetes, Obesity and Metabolism. 2017;19(12):1706-13.

30. Lindman BR, Davila-Roman VG, Mann DL, McNulty S, Semigran MJ, Lewis GD, et al. Cardiovascular phenotype in HFpEF patients with or without diabetes: a RELAX trial ancillary study. Journal of the American College of Cardiology. 2014;64(6):541-9.

31. Eitel I, Hintze S, De Waha S, Fuernau G, Lurz P, Desch S, et al. Prognostic impact of hyperglycemia in nondiabetic and diabetic patients with ST-elevation myocardial infarction: Insights from contrast-enhanced magnetic resonance imaging. Circulation: Cardiovascular Imaging. 2012;5(6):708-18.

32. Donnino R, Patel S, Nguyen AH, Sedlis SP, Babb JS, Schwartzbard A, et al. Comparison of quantity of left ventricular scarring and remodeling by magnetic resonance imaging in patients with versus without diabetes mellitus and with coronary artery disease. American Journal of Cardiology.

2011;107(11):1575-8.

Page $7 / 14$ 
33. Ramos R, Albert X, Sala J, Garcia-Gil M, Elosua R, Marrugat J, et al. Prevalence and incidence of Q-wave unrecognized myocardial infarction in general population: Diagnostic value of the electrocardiogram. The REGICOR study. International journal of cardiology. 2016;225:300-5.

34. Barbier CE, Bjerner T, Johansson L, Lind L, Ahlström H. Myocardial scars more frequent than expected: magnetic resonance imaging detects potential risk group. Journal of the American College of Cardiology. 2006;48(4):765-71.

35. Bertoni AG, Goff Jr DC, D'Agostino Jr RB, Liu K, Hundley WG, Lima JA, et al. Diabetic cardiomyopathy and subclinical cardiovascular disease: The MultiEthnic Study of Atherosclerosis (MESA). Diabetes Care. 2006;29(3):588-94.

36. Shivu GN, Phan TT, Abozguia K, Ahmed I, Wagenmakers A, Henning A, et al. Relationship between coronary microvascular dysfunction and cardiac energetics impairment in type 1 diabetes mellitus. Circulation. 2010;121(10):1209-15.

37. Campbell DJ, Somaratne JB, Jenkins AJ, Prior DL, Yii M, Kenny JF, et al. Impact of type 2 diabetes and the metabolic syndrome on myocardial structure and microvasculature of men with coronary artery disease. Cardiovascular diabetology. 2011;10:80.

38. Tarquini R, Lazzeri C, Pala L, Rotella CM, Gensini GF. The diabetic cardiomyopathy. Acta diabetologica. 2011;48(3):173-81.

39. Gao Y, Yang ZG, Ren Y, Liu X, Jiang L, Xie LJ, et al. Evaluation of myocardial fibrosis in diabetes with cardiac magnetic resonance T1-mapping: Correlation with the high-level hemoglobin A1c. Diabetes research and clinical practice. 2019;150:72-80.

40. Turkbey EB, Backlund JY, Genuth S, Jain A, Miao C, Cleary PA, et al. Myocardial structure, function, and scar in patients with type 1 diabetes mellitus. Circulation. 2011;124(16):1737-46.

41. Ng ACT, Strudwick M, van der Geest RJ, Ng ACC, Gillinder L, Goo SY, et al. Impact of Epicardial Adipose Tissue, Left Ventricular Myocardial Fat Content, and Interstitial Fibrosis on Myocardial Contractile Function. Circ Cardiovasc Imaging. 2018;11(8):e007372.

42. Anselmino M, Matta M, D'Ascenzo F, Pappone C, Santinelli V, Bunch TJ, et al. Catheter ablation of atrial fibrillation in patients with diabetes mellitus: a systematic review and meta-analysis. Europace. 2015;17(10):1518-25.

43. Gulsin GS, Kanagala P, Chan DCS, Cheng ASH, Athithan L, Graham-Brown MPM, et al. Differential left ventricular and left atrial remodelling in heart failure with preserved ejection fraction patients with and without diabetes. Therapeutic Advances in Endocrinology and Metabolism. 2019;10(2042018819861593).

44. Mordi I, Bezerra H, Carrick D, Tzemos N. The Combined Incremental Prognostic Value of LVEF, Late Gadolinium Enhancement, and Global Circumferential Strain Assessed by CMR. JACC Cardiovascular imaging. 2015;8(5):540-9.

45. Sandesara PB, O'Neal WT, Kelli HM, Samman-Tahhan A, Hammadah M, Quyyumi AA, et al. The Prognostic Significance of Diabetes and Microvascular Complications in Patients With Heart Failure With Preserved Ejection Fraction. Diabetes Care. 2018;41(1):150-5.

46. Schelbert EB, Cao JJ, Sigurdsson S, Aspelund T, Kellman P, Aletras AH, et al. Prevalence and prognosis of unrecognized myocardial infarction determined by cardiac magnetic resonance in older adults. Jama. 2012;308(9):890-6.

47. Acharya T, Aspelund T, Jonasson TF, Schelbert EB, Cao JJ, Sathya B, et al. Association of Unrecognized Myocardial Infarction With Long-term Outcomes in Community-Dwelling Older Adults: The ICELAND MI Study. JAMA Cardiol. 2018;3(11):1101-6.

48. Cao Y, Zeng W, Cui Y, Kong X, Wang M, Yu J, et al. Increased myocardial extracellular volume assessed by cardiovascular magnetic resonance T1 mapping and its determinants in type 2 diabetes mellitus patients with normal myocardial systolic strain. Cardiovascular diabetology. 2018;17(1):7.

49. Wang H, Ba Y, Cai R-C, Xing Q. Association between diabetes mellitus and the risk for major cardiovascular outcomes and all-cause mortality in women compared with men: a meta-analysis of prospective cohort studies. BMJ open. 2019;9(7):e024935.

50. Dabelea D, Stafford JM, Mayer-Davis EJ, D'Agostino R, Jr., Dolan L, Imperatore G, et al. Association of Type 1 Diabetes vs Type 2 Diabetes Diagnosed During Childhood and Adolescence With Complications During Teenage Years and Young Adulthood. Jama. 2017;317(8):825-35.

\section{Figures}




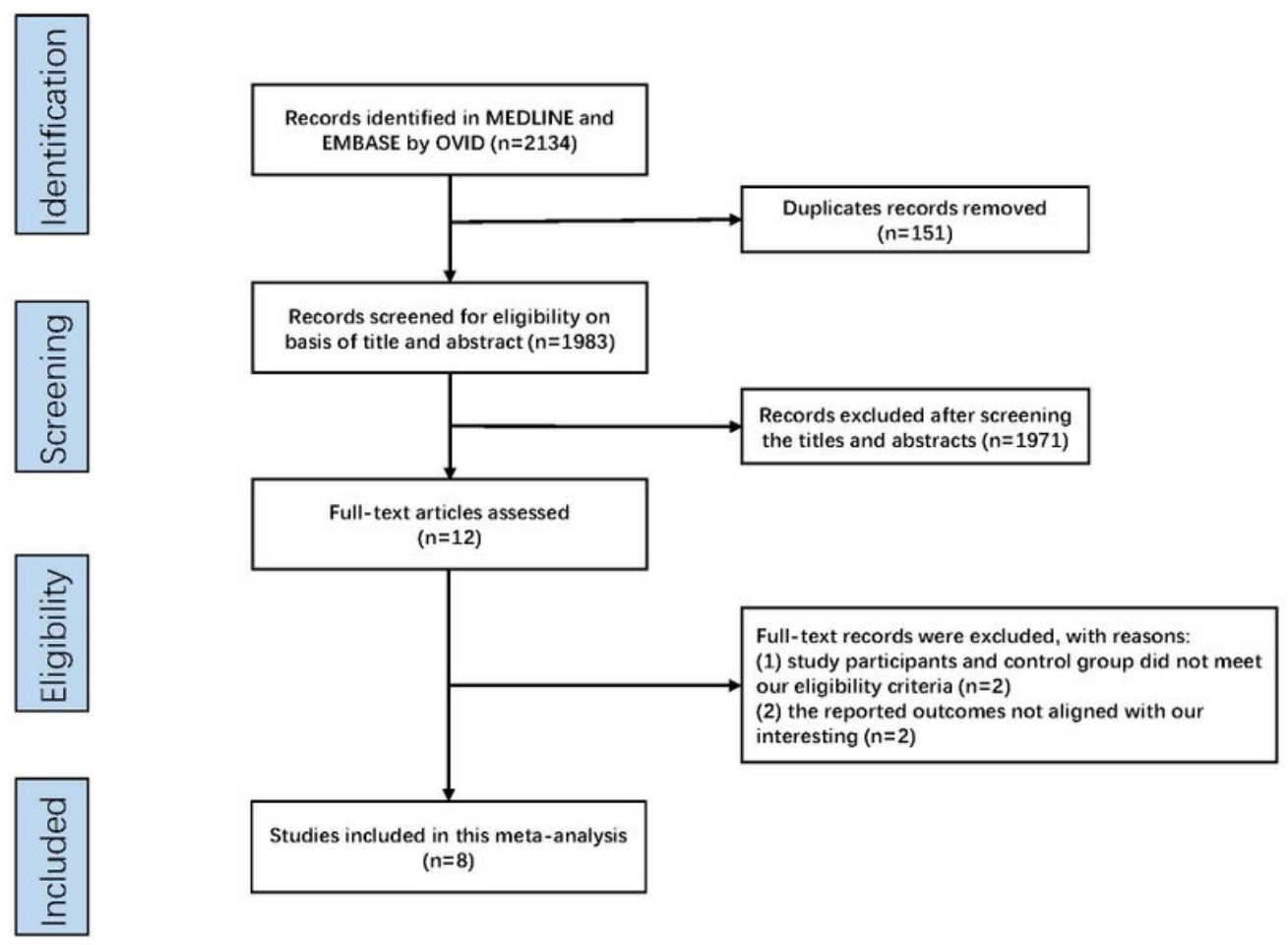

Figure 1

Flow chart of literature and study selection. 
Funnel plot with pseudo $95 \%$ confidence limits

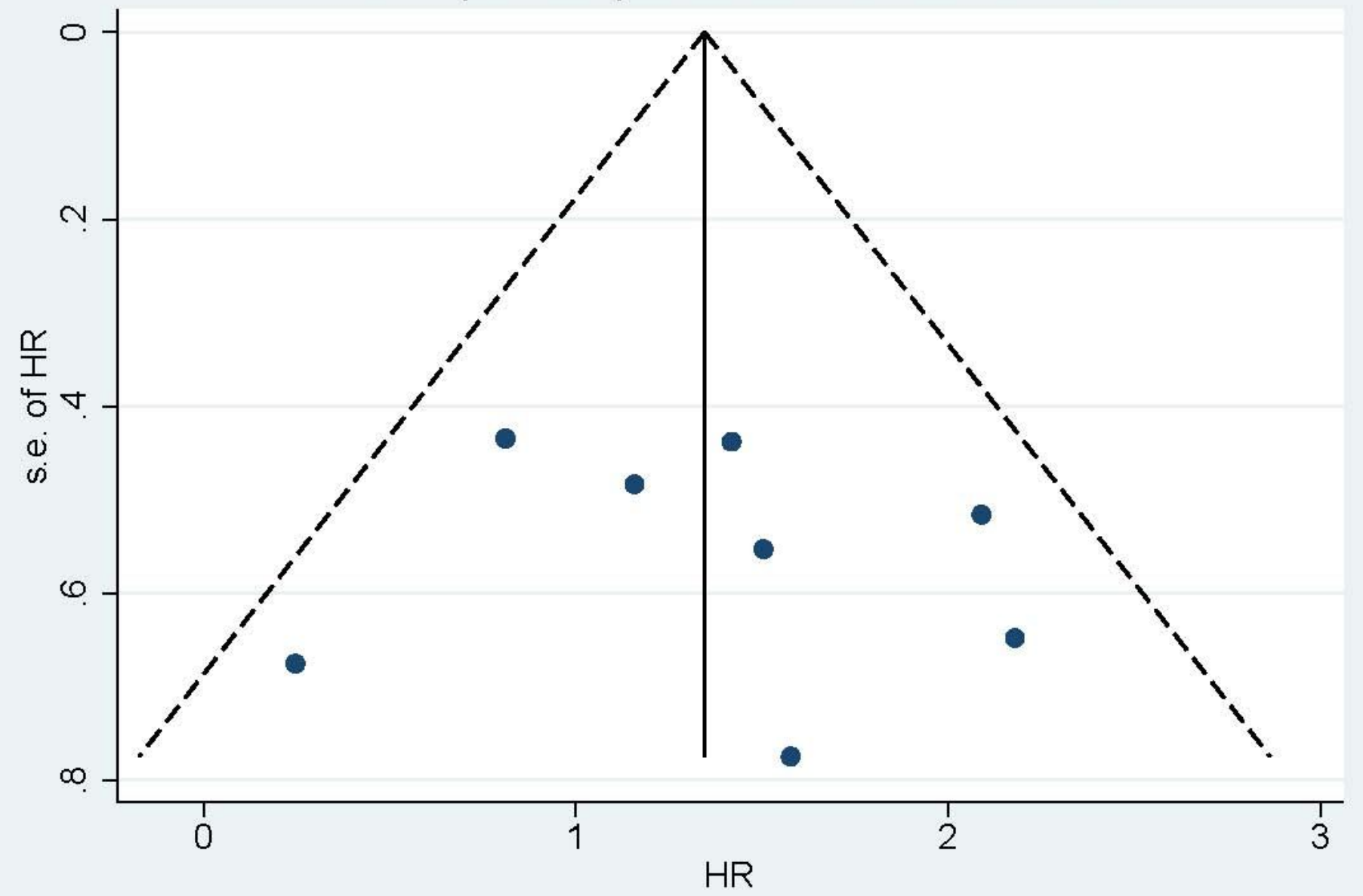

Figure 2

Funnel plots of 8 eligible studies. 
Study

ID
$\%$

HR $(95 \% \mathrm{Cl}) \quad$ Weight

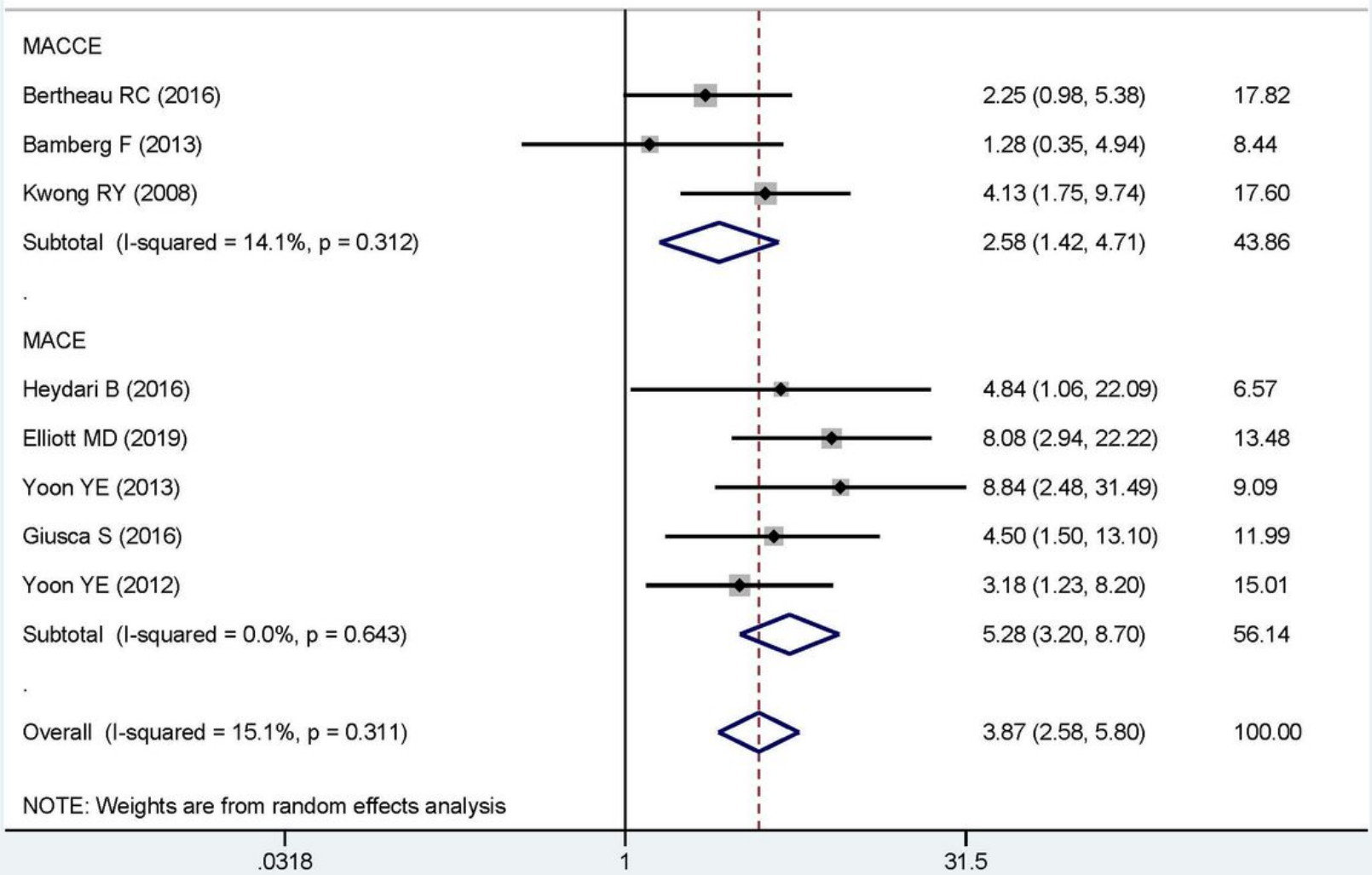

\section{Figure 3}

Forest plot and pooled estimates of the effect of myocardial fibrosis detected by LGE on the risk of MACCE or MACE. LGE, late gadolinium enhancement; MACCE, major adverse cardiac and cerebrovascular events; MACE, major adverse cardiac events; HR, hazard ratios; $\mathrm{Cl}$, confidence interval. 
Study

ID
$\%$

HR $(95 \% \mathrm{Cl}) \quad$ Weight

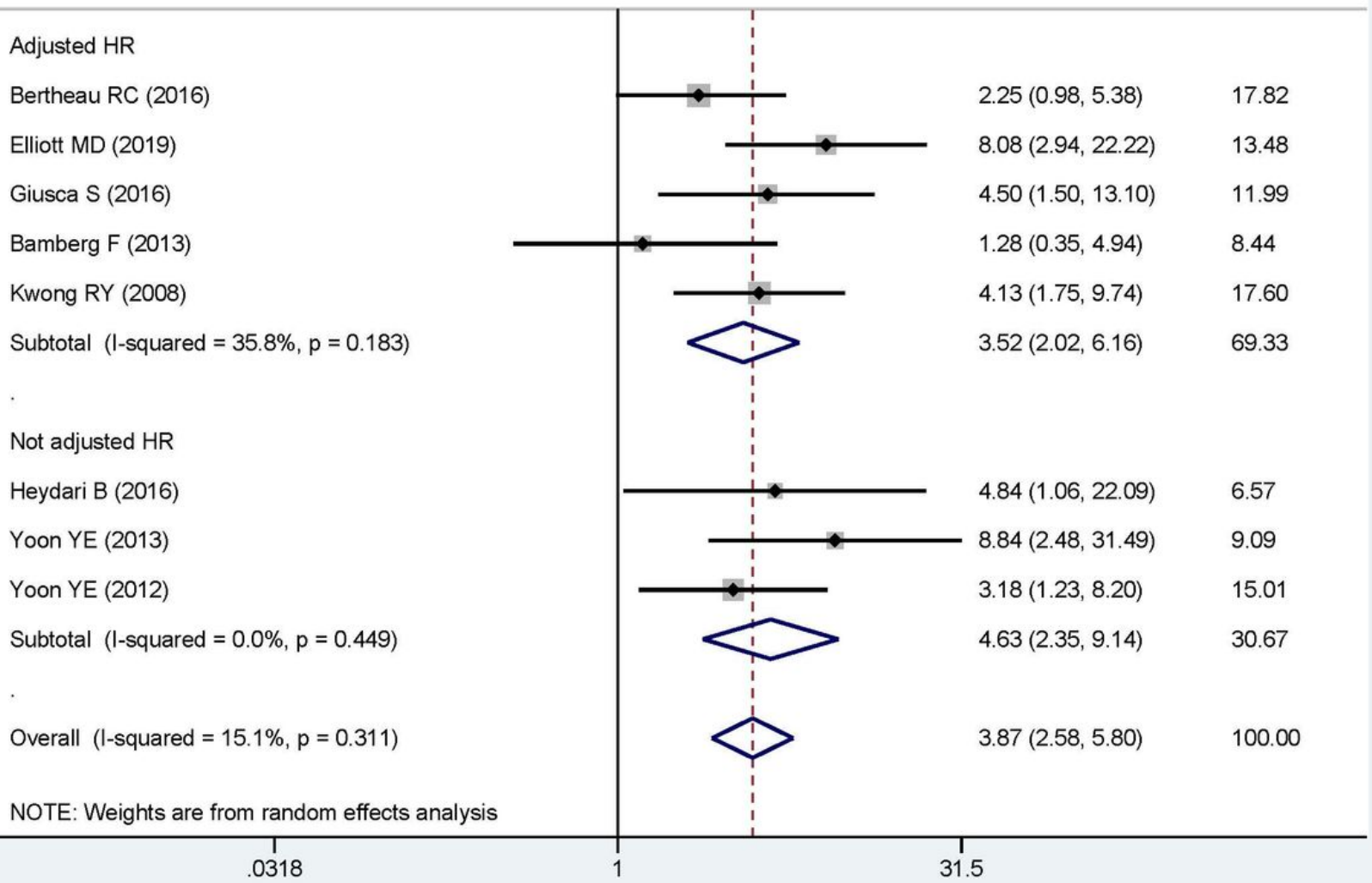

\section{Figure 4}

Forrest plots of pooled HR for MACCE and MACE in adjusted or not adjusted HR studies. HR, Hazard Ratios; MACCE, major adverse cardiac and cerebrovascular events; MACE, major adverse cardiac events; HR, Hazard Ratios; Cl, confidence interval. 
Study

ID
$\%$

$\mathrm{HR}(95 \% \mathrm{Cl}) \quad$ Weight

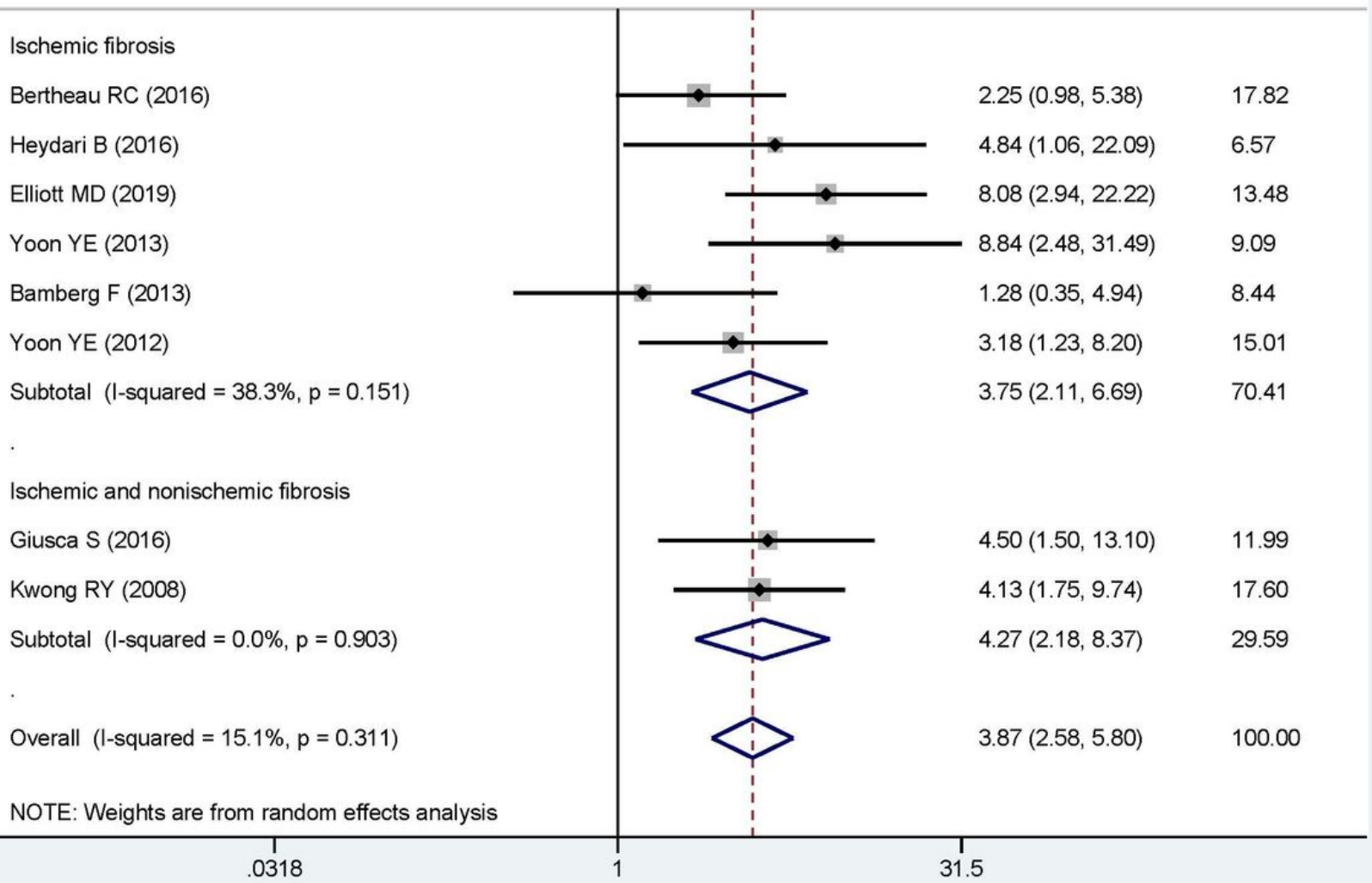

\section{Figure 5}

Forrest plots of selected studies for pooled HR for MACCE and MACE in patients with diabetes and different pattern of myocardial fibrosis detected by LGE. HR, Hazard Ratios; LGE, late gadolinium enhancement; MACCE, major adverse cardiac and cerebrovascular events; MACE, major adverse cardiac events; Cl, confidence interval. 


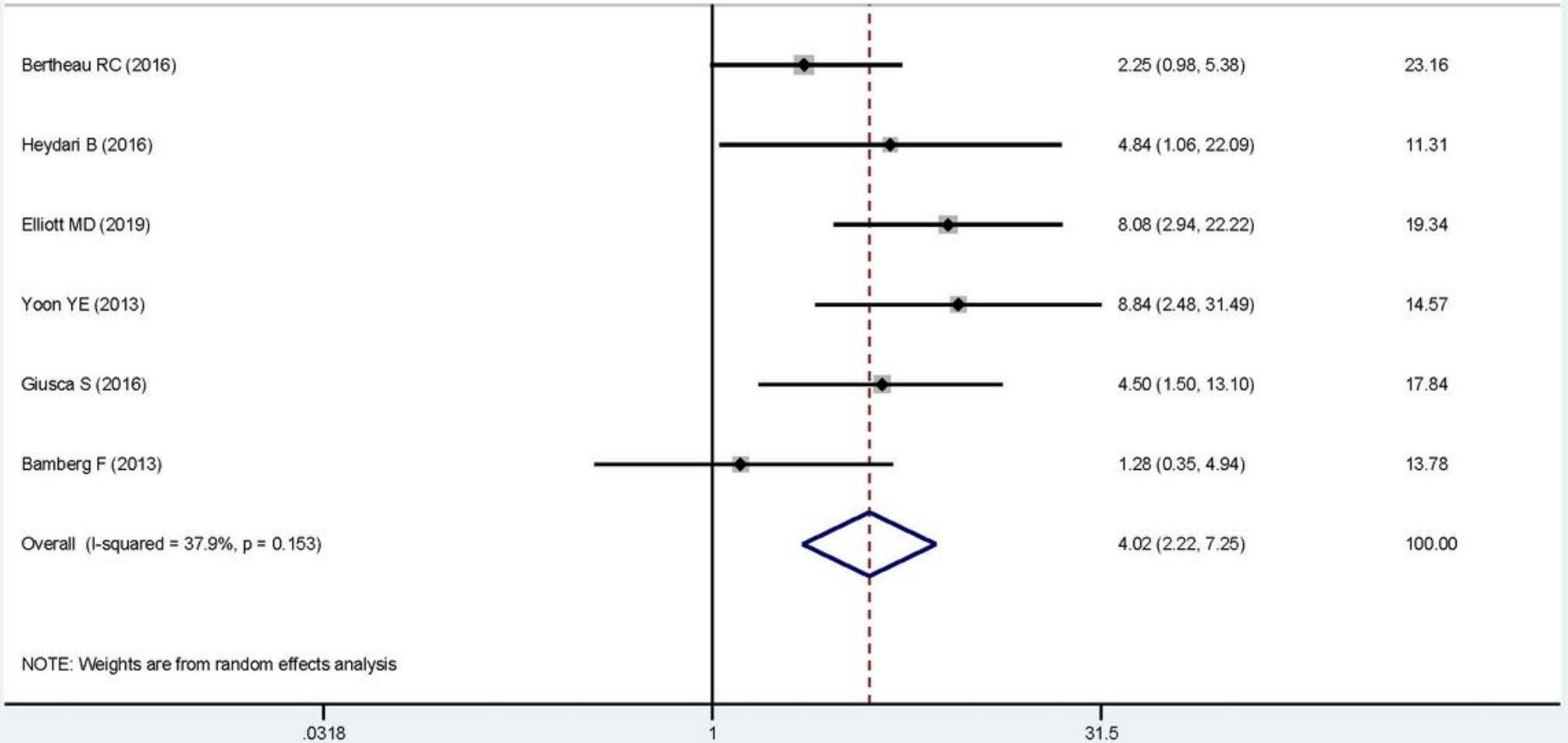

\section{Figure 6}

Forrest plots of 6 studies for pooled HR for MACCE and MACE in patients with diabetes with normal LV ejection fraction and myocardial fibrosis detected by LGE. HR, Hazard Ratios; LGE, late gadolinium enhancement; MACCE, major adverse cardiac and cerebrovascular events; MACE, major adverse cardiac events; $\mathrm{Cl}$, confidence interval.

\section{Supplementary Files}

This is a list of supplementary files associated with this preprint. Click to download.

- SupplementTabeS1.docx

- SupplementTabeS1.docx 